\title{
Ultrasonography: use of echo-doppler in identification of endoleaks in post-implantation of thoracic endovascular aortic repair (TEVAR)
}

\begin{abstract}
Introduction: A thoracic aortic aneurysm (TAA) is a condition with a high mortality rate, which is associated with atherosclerosis, age, infection, inflammation, trauma, congenital abnormality or degeneration. The main treatment is conventional Surgery and there is an emerging technique, Thoracic Endovascular Aortic Repair (TEVAR). The latter is a noninvasive procedure, quickly adopted by clinicians for the endovascular treatment of TAA.

Purpose: The purpose of this review article is to highlight the advantages and the appropriateness of the use of Doppler ultrasound in the early detection and follow-up of endoleaks after TEVAR compared with other imaging techniques.

Methods: We conducted a review of the literature, based on the keywords: aneurysm, TEVAR, endoleak, follow-up, imaging techniques, Doppler ultrasound. We used PubMed and B-on data, where 48 articles were selected, 27 of these were analyzed because they corresponded to the keywords above.

Development: The main complication inherent in TEVAR, is the formation ofendoleaks. Thus, it is extremely important to employ different imaging techniques. Computerized tomography (CT) is the Gold Standard in the detection and identification of endoleaks. Doppler ultrasound has been recognized as a good choice for this technique due to its low cost, easy interpretation and implementation and lack of exposure to any type of radiation.

Conclusion: Doppler ultrasound is an imaging technique of high quality and comparable to $\mathrm{CT}$ in the measurement of aneurysm, endoleak after TEVAR identification and can be used for surveillance and follow-up.
\end{abstract}

Keywords: aneurysm, tevar, endoleak, follow up, imaging techniques, doppler ultrasound
Volume I Issue 4 - 2014

\author{
Lia Marques,' Ângela Martins,' Paulo \\ Batista $^{1,2}$ \\ 'Lisbon School of Health Technology (ESTeSL), Portugal \\ ${ }^{2}$ Lisbon Centre North Hospital, EPE, Portugal
}

Correspondence: Lia Marques, Lisbon School of Health Technology (ESTeSL), Rua Machado Santos, n 3, 2540-I I 4 Bombarral, Portugal, Email liammarques92@gmail.com

Received: July 21, 2014 | Published: October 10, 2014
Abbreviations: ATT, a thoracic aortic aneurysm; TEVAR, thoracic endovascular aortic repair; CT, computerized tomography; $\mathrm{CAD}$, coronary artery disease; RF, Renal Failure; PET, positron emission tomography; PD, power doppler

\section{Aneurysm}

The TAA is a significant life-threatening condition, causing mortality in the short and long term due to its rupture and dissection. It is defined as the dilation of the aorta $50 \%$ higher than the normal diameter of the same in a given segment. ${ }^{1}$ Aortic aneurysms may be classified according to their morphology, etiology and location. In the morphological classification we have fusiform and saccular aneurysms. The ones with fusiform shape are cylindrical and affect the entire circumference of the aorta and the saccular form has an intussusception portion of the aortic wall. Saccular aneurysms have a greater rupture risk than fusiform aneurysms. ${ }^{2-4}$

TAA's are mostly saccular, with a small connecting canal between the cavity of the aneurysm and the aortic lumen. This restricted orifice protects the thin wall of the aneurysm from the intra-aortic pressure, thus reducing the risk of breakage, in contrast with the fusiform aneurysm. Aneurysms can also be classified according to the involved segment, thoracic, thoracoabdominal or abdominal. ${ }^{2,5}$ TAA's are distributed equally in men and women, increasing in frequency with advancing age. They are sometimes associated with hereditary factors which involving connective tissue, including Marfan syndrome (Figure $1 \& 2$ )..$^{5,6}$
Smooth muscle cells from patients with aortic aneurysms, as compared to the normal aortas have proliferative properties. The increase of this migration, which seems to be due to overproduction of matrix metalloproteinases (MMP-2) may lead to the formation of aneurysms. An abnormal active biochemical activity, elastolytic and proteolytic, was identified in tissues of the aortic aneurysm. Abnormal macrophages and increased levels of cytokines in the same tissue is also indicative of an active inflammatory process which may contribute to the pathogenesis of this. The smooth muscle cells developed from the aneurysm compared to normal cells, produce high levels of plasminogen activators.

This can increase the amount of plasmin available for the zymogen activation of MMP-2, thus favoring proteolysis in the absence of a bilateral increase in plasmin activator inhibitor. MMP-2 may therefore play an important role in the degeneration of the aortic wall, which leads to the formation of aneurysm. ${ }^{9}$ The weakening of the aortic wall is exacerbated by an increase of systolic pressure, especially in the ascending aorta. This segment of the aorta is most exposed to the pressure of each cardiac systole and the dynamic movement of the heart transmitted every cardiac cycle. The weakening of local walls causes aortic dilation and increase wall tension (described by the Laplace Law). Small cracks in the intimal layer of the aorta may allow blood to penetrate the middle layer, leading to a dissection. ${ }^{1}$ The aortic dilation can occur as a result of atherosclerosis, increase in age, infection, inflammation, trauma, congenital abnormalities or degeneration. ${ }^{2,5}$ 


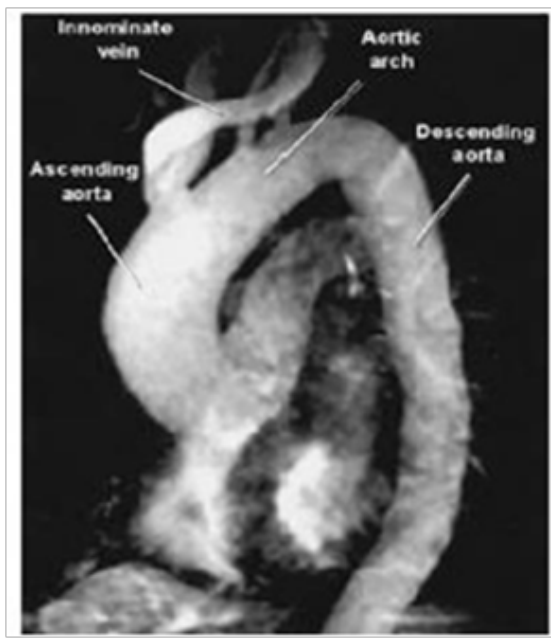

Figure I MRI angiography demonstrates ascending TAA. ${ }^{7}$

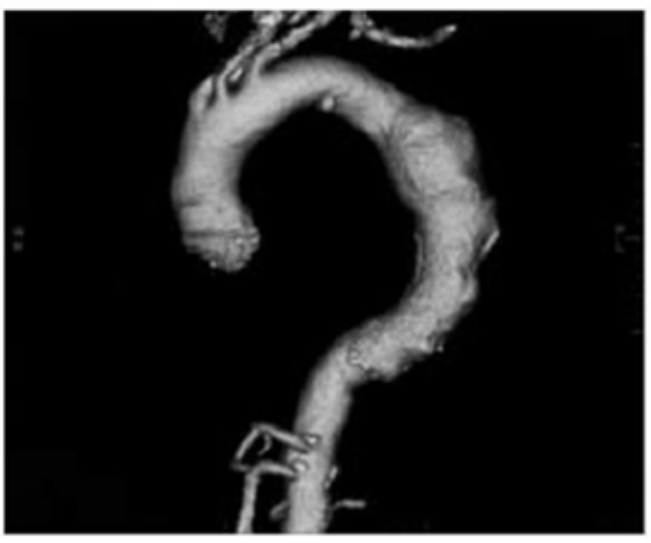

Figure 2 CT angiography demonstrating TAA ${ }^{8}$

\section{Conventional surgery}

Conventional surgery is the most common method in the treatment of TAA. This involves median sternotomy (for aneurysms of the ascending aorta (Asc. Ao) and aortic arch) or left thoracotomy (for aneurysms of the descending aorta (Desc. Ao) and thoracoabdominal) and subsequent excision of the aneurysm, replacing it with a synthetic graft. This surgery is indicated for patients with large aneurysms (diameter $>5-6 \mathrm{~cm}$ in Asc. Ao; $>$ At $6-7 \mathrm{~cm}$ in Desc. Ao and $>5 \mathrm{~cm}$ in Marfan Syndrome patients at any location), aneurysms that grow rapidly $(>1 \mathrm{~cm} /$ year $)$ and symptomatic, traumatic or syphilitic aneurysms.

Although surgical repair of an intact TAA improves the prognosis, the mortality rate may still exceed 5 to $10 \%$ in 30 days and reach 40 to $50 \%$ at 10 years. The risk of death increases if aneurysms develop complications (eg. the aortic arch or thoracoabdominal aorta), if patients have Coronary Artery Disease (CAD), Renal Failure (RF) preexisting with advanced age or if they are symptomatic. Perioperative complications (eg. cerebrovascular accident (stroke), spinal injury and RF) occurs in about 10 to $20 \%$ of cases. ${ }^{9}$

\section{TEVAR}

TEVAR is an Emerging method of treatment that has been rapidly adopted by clinicians in the treatment of thoracic aortic conditions. ${ }^{10}$ The range of this disease includes aneurysms, acute and chronic dissection of the descending thoracic aorta, penetrating ulcer, intramural hematoma and traumatic rupture. ${ }^{11,12}$ The definite indications for intervention are rupture and symptomatic aortic disease. In asymptomatic patients with thoracic aneurysm the decision to intervene is complex and clinicians should consider the risk of operability versus the risk of rupture and death without treatment.

TEVAR is a less invasive technique compared to conventional surgery and its availability and relative ease of application, amplified and changed the options in the management of thoracic aortic disease, including patients considered unfit for conventional surgery (Figure 3$).{ }^{10}$

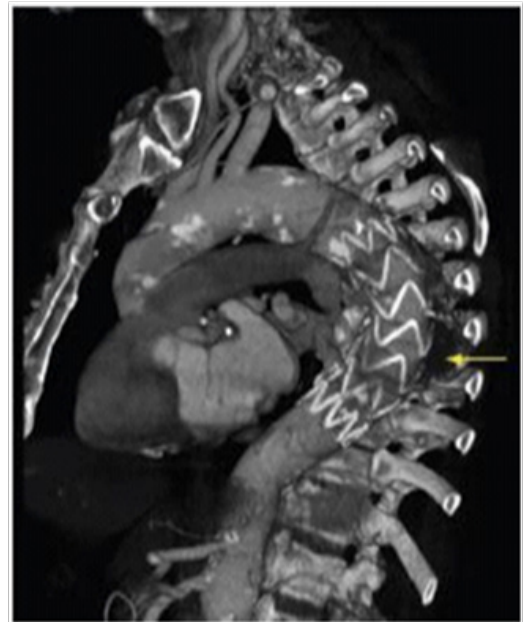

Figure 3 CT Angiography after TEVAR. ${ }^{10}$

The main purpose of endovascular treatment of aneurysmal disease is to eliminate existing blood flow into the aneurysmal sac, internally connecting the arterial lumen and stabilizing the proximal and distal area of the dilated segment to prevent rupture of the aneurysm. ${ }^{13}$ This proceeds to the deployment of endovascular stents through catheters, which can also be used in combination with conventional surgery on the thoracic aorta, for example, in diffuse aortic disease. ${ }^{11}$

The large and rapid expansion of the use of this technique has a systematic requirement concerning indications, suitability and limitations, since it has been adopted by many specialties, including cardiologists, cardiovascular surgeons, radiologists and vascular surgeons. It is extremely important to recognize the anatomy of the aorta and its need for adequate vascular access. The choice of patients with inadequate anatomy must be prevented by a proper selection of them. ${ }^{14}$ There are three indications for placement of TEVAR: individuals with TAA, with aortic dissection and traumatic injury. For effective placing an attachment region with atleast $2 \mathrm{~cm}$ is required, either proximal or distal to obtain a secure deployment and lasting fixation of TEVAR. ${ }^{10}$

There are many risk factors that may influence the implantation of TEVAR, particularly, the combination of the presence of atherosclerotic walls and tortuosity observed in the thoracic aorta of atherosclerotic aneurysms. This may require a change in the fixation of the stent in the course of time due to the relative stiffness of the whole fixing system. ${ }^{14}$ Apart from these factors, there are inherent complications in this procedure, such as stent migration, aneurysm growth and the formation of endoleaks.

It is known that the type of image obtained and intervals of followup after aneurysms repair are topics of discussion, Appropriate image 
monitoring being extremely important to detect post-procedure complications. The formation of endoleaks is the most common complication of this procedure, as such, it is of utmost importance for the condition to be monitored through various imaging techniques. ${ }^{15}$

\section{Endoleak}

Endoleak is related to endovascular grafts, defined by the presence of blood flow outside the lumen of stent graft but within the aneurysm $\mathrm{sac}$ or at the adjacent segment that is being treated with the graft. It is due to an incomplete sealing, of the aneurysmal sac or a segment of the vessel identified by various imaging techniques such as CT, ultrasound or angiography. ${ }^{15}$

Some endoleaks appear to be an inevitable consequence of placing a stent graft inside the aneurysm sac, with vessels for the outflow, while others are the result of a wrong selection of the stent, or because the patient is unfit for this type of procedure ${ }^{16}$. The occurrence of endoleaks depends on the material used and the experience of the operating team in patient selection and implantation techniques. The use of the latest materials and equipment will decrease the rate of complications in the future. ${ }^{13}$

According with Ashoke et al. ${ }^{17}$ endoleaks and other complications occur in about a quarter or more of all patients subject to the TEVAR deployment, without a decrease over time. In Heilberger et al. ${ }^{18}$ a study in a population with 108 men with a mean age of 67.3 years, the incidence of endoleaks after TEVAR ranged between $2.4 \%$ and $44 \%$. Moreover, according to Chagas Neto et al. ${ }^{19}$ with a sample of 20 cases to illustrate the different types of endoleaks, we verified the existence of endoleaks in between $10 \%$ to $25 \%$ of cases, with spontaneous resolution in only $40 \%$ to $50 \%$ of them. Thus, a continuous vigilance for visualization, evolution and early identification of the type of endoleak, is required.

The endoleak may be classified according to the time it is detected. During the perioperative period ( $<30$ days) it is defined as a primary endoleak. If after that time, it will be designated as a secondary endoleak..$^{20}$ In addition to this temporal classification, there is another based on their genesis, divided into five types:

1) Type I endoleaks are defined by the stent detachment in certain locations of the vessel wall, in a proximal (type Ia) or distal (type $\mathrm{Ib}$ ) level. The blood flow, which should pass through the stent in its entirety, is diverted into the space between the stent graft and the aneurysm wall. This flow is subjected to systemic pressure, and leads to the risk of the aneurysmal sac expanding and causing it to rupture. These are always considered clinically significant, and are generally treated, once they are detected, since its spontaneous resolution is impossible.

2) Type II endoleaks are defined by the presence of retrograde flow in collateral vessels into the space between the stent and the vessel wall, thereby predicting the aneurysmal sac thrombosis and creating a risk of progressive expansion and possible rupture thereof.

3) The type III endoleaks are defined by the failure or disjunction of the components of the modular system of the graft or a manufacturing defect of the same. There is a direct entry of blood flow to the space between the graft and the vessel wall, such as in the Type I endoleaks, where an intervention is needed.

4) The type IV endoleaks are not a real leak but represent the passage of blood or fluid through the porosity of the graft itself. This type of endoleak ceased to exist due changes made at the level of porosity in the manufacture of grafts.
5) Finally, type $V$ endoleaks are defined as the continued increase in size of the aneurysm sac without any visible leakage of blood flow into the aneurysm sac as seen through various imaging techniques.

Is also referred as endotension, being a poorly understood phenomenon, but it is suspected that it is due to the pulsation of the graft wall, where a transmission by the space between it and the aneurysm wall occurs; ultrafiltration of the blood through the graft or the presence of a thrombus, which also symbolizes an ineffective barrier to pressure transmission (Figure 4) ${ }^{16,21}$ It appears that there is a group of patients with a greater predisposition to endoleaks formation after TEVAR. According to Parmer et al..$^{22}$ patients at higher risk of developing endoleaks after the intervention are those with a larger and more extensive aneurysm, as well as those who need a coverage of the aorta with multiple stents. Data from this study also suggest that the use of long devices rather than short multiple devices can provide superior results, reducing the incidence of endoleaks after an

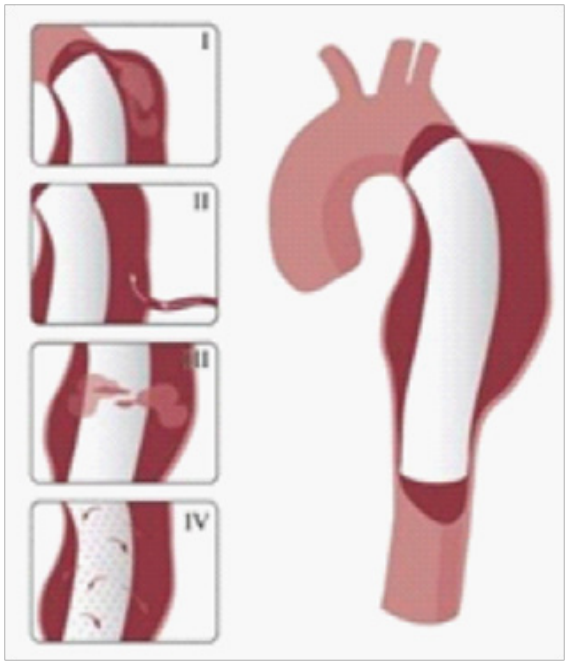

intervention

Figure 4 Endoleak types. ${ }^{8}$

Endoleaks will compromise the stent graft function (total isolation of the aneurysmal sac thus reducing the pressure inside this which leads to a shrinkage of the aneurysm), so their detection is important in the analysis of clinical success after endovascular aneurysm correction. ${ }^{23}$ All of this involves strategies for the treatment of endoleaks that must be followed according to the degree of development and repercussions from these:

a) Conservative observation with monitoring through image repetition;

b) Correction with an additional endovascular graft;

c) Embolization and other endovascular interventions;

d) Surgical placement of a ligature of the aortic neck band;

e) Conversion to conventional aneurysm repair. ${ }^{13}$

\section{Follow-up}

The image type and intervals of follow-up after TEVAR are topics under discussion, it being clear that vigilance is needed through imaging techniques to detect post procedure complications, to indicate later, the most appropriate treatment to be offered for the best result for patients. ${ }^{15,22}$ These techniques will detect endoleaks, will monitor 
the size of the aneurysmal sac and will confirm the setting and position of the graft. ${ }^{17,24}$ According with Heilberger et al. ${ }^{18}$ the follow-up examinations should be performed three to six months apart, always considering the clinical course of each patient. Imaging techniques used after endovascular aneurysm correction will influence the rate of endoleaks reported. As such, a technique is required with higher sensitivity in the detection of these, which may be useful for further patient treatment. ${ }^{23}$

\section{Imaging techniques}

There are several techniques for the detection of endoleaks after endovascular aortic treatment such as X-ray, Angiography by Computerized Tomography (CT angiography), Angiography by Magnetic Resonance (MRI angiography), aortography, ultrasound and others, less used. ${ }^{13,23,25} \mathrm{An} \mathrm{X}$-ray allows a view of the mediastinum widening on the left and an enlargement of Asc and Dsc. Ao. However, it does not distinguish the tortuosity level of the aneurysm in the aorta, which causes some not to be detected. The CT angiography is effective in determining the location, size and/or any complications. The main advantages being its high reproducibility for follow-up studies and early detection of complications such as perforation with hematoma in the mediastinum, pleural effusion, pericardial effusion and signs of aortic syndrome. The disadvantages are that we have the use of contrast agents and an inability to visualize aortic regurgitation and motion abnormalities of the left ventricular wall. The MRI angiography produces panoramic high-resolution views of the aorta in cross section, sagittal and coronal planes, providing the ideal source and delineation of the entire length of the aneurysm. A low emission of radiation is the most important advantage. However, the continued use of contrast is the main drawback, mainly affecting renal failure, with a high risk of developing Renal Systemic Fibrosis.

Aortography is an invasive technique to visualize aortic regurgitation, location, size and extent of the aneurysm, assess ventricular function and coronary artery disease. The main disadvantage is an exposure to radiation and the use of contrast agents. Finally, ultrasound, to visualize the entire aorta using transesophageal, suprasternal, subcostal and abdominal echocardiography. This has disadvantages in the fact that the ascending part of the aortic arch cannot be viewed even by transesophageal echocardiography. It's a place with difficult access, so it is not very effective in determining the extent and full size of the aneurysm. Although not widely used, there are other techniques for endoleaks detection. Among these we can highlight the Intravascular ultrasound using probes from 7 to $10 \mathrm{MHz}$, mechanical and electronic scanners that show crosssectional images, the Positron Emission Tomography (PET) to detect increased metabolic activity in the thoracic and abdominal aneurysms, fluorodeoxyglucose uptake (the aneurysm level is a strong predictor of dilation and rupture of the same) and finally laboratory tests that help identify the inflammatory activity. The most commonly used techniques are CT and MRI angiography, allowing a good view of the aneurysmal sac with excellent resolution and after contrast injection, to determine the location, size and/or any complications. ${ }^{25}$ The endoleak size is an important factor for detection, since it influences their detection. ${ }^{23}$ However, these techniques have disadvantages such as radiation exposure and potential nephrotoxicity and its high cost. ${ }^{11,26}$ These are less sensitive to detection of contrast outside the lumen, making it less visible with contrast dilution, causing an underestimation of the endoleaks. ${ }^{23}$

In contrast, Echo Doppler has been recognized as a good option to these techniques due to its low cost, easy implementation and interpretation and the absence of exposure to any type of radiation. ${ }^{26}$ This provides a better understanding of the endoleak formation mechanism and provides a more accurate analysis thereof, due to a collateral circulation. The origins of an endoleak may also be correctly identified. Compounded by the fact that it allows visualize blood flow in the aneurysm sac and its direction. This seems to be an excellent tool in the evaluation of high flow endoleaks within the aneurysmal sac. It is an imaging technique commonly used in vascular studies, being easy to perform, inexpensive, portable, secure and highly available. Its use for patients' follow-up can reduce the biological hazards associated with CT angiography, since this frees the body of the individual to a substantial dose of ionizing radiation. The advantage of Doppler ultrasound in patients' follow-up with aortic stent graft is that it allows a serial measurement over time of the residual diameter of the aorticaneurysm sac. Doppler ultrasound is more accurate than CT for the detection of problems associated with stent graft patency, their migration, kinking and stenosis. Provides physiological and anatomical information at the same time, unlike CT. The ability to quantify and compare serial images at a low cost without contrast and radiation, suggests that this technique should be the Gold Standard in the follow-up to TEVAR (Figures 5-9). ${ }^{20,24,2}$

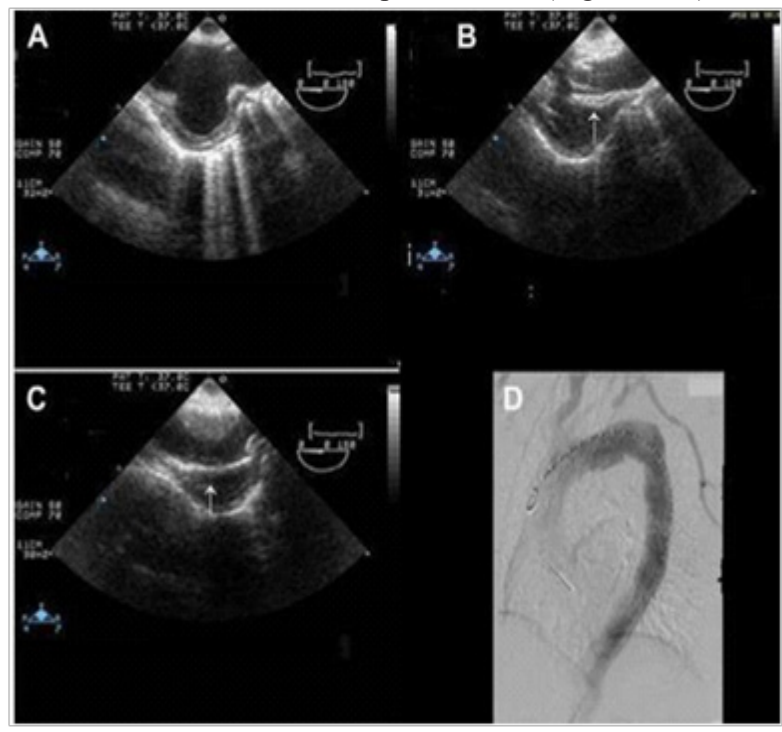

Figure 5 Image showing (A) arch aneurysm, (B) partially deployed stent graft (arrow), and (C) fully deployed stent graft (arrow). (D) Post procedure angiography showing stent grafts in place..$^{28}$

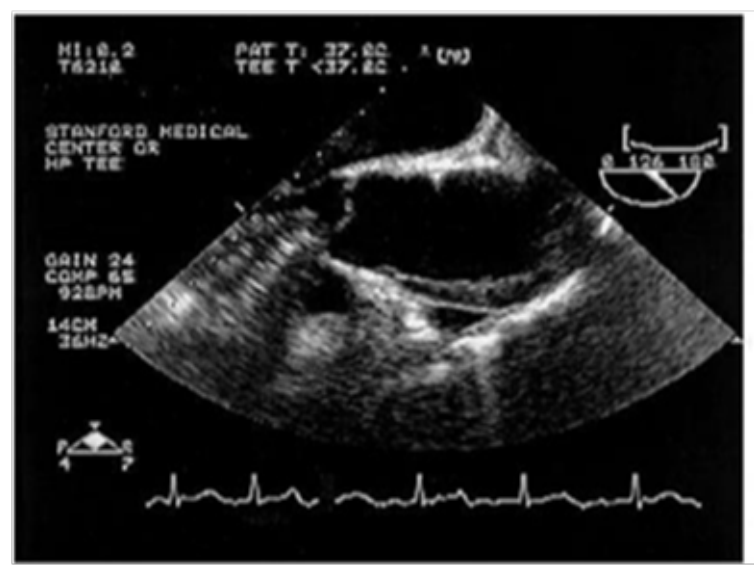

Figure 6 Ascending aorta showing intramural hematoma of the anterior wall of the ascending aorta. ${ }^{29}$ 


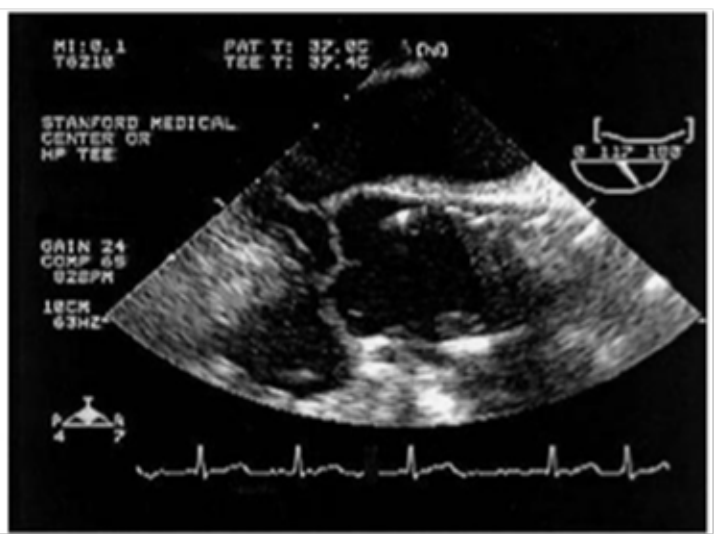

Figure 7 Ascending aorta showing the position of the stent after deployment. ${ }^{29}$

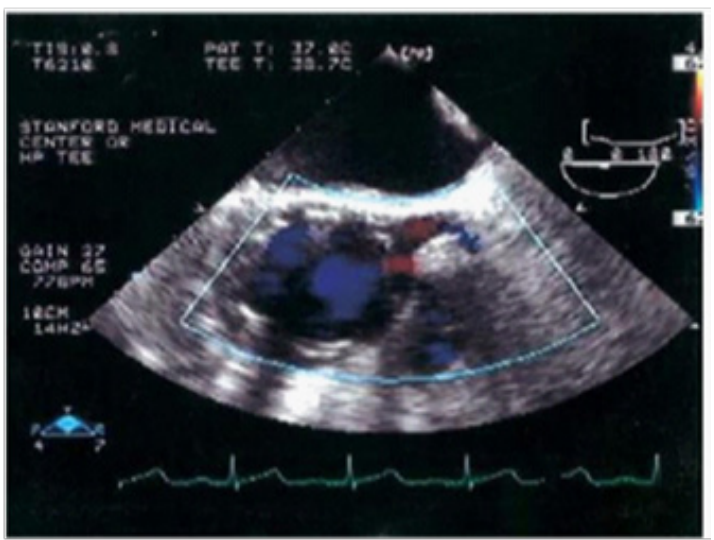

Figure 8 Ascending aorta/aortic root. The image shows the position of the graft and the patency of the left main coronary artery after the deployment of the graft. ${ }^{29}$
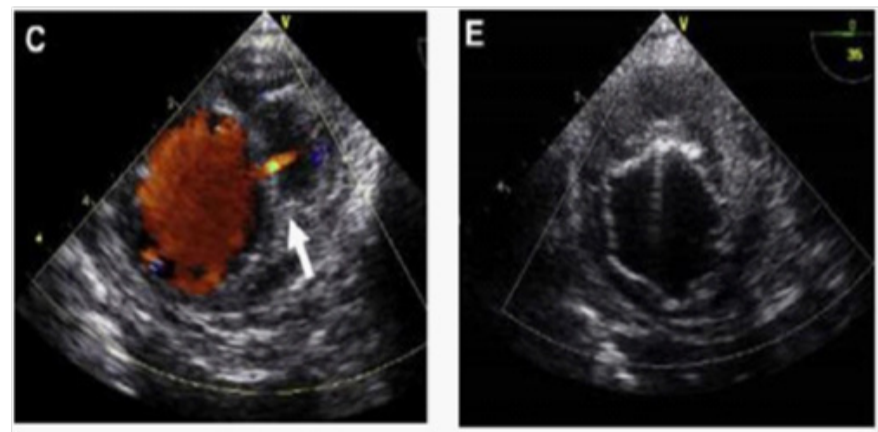

Figure 9 (C) Image showing active bleeding from the aortic lumen into the hematoma (arrow). (E) TEVAR showing elimination of flow. ${ }^{30}$

\section{Image protocol}

The use of Echo-Doppler follow-up requires a protocol to be followed that includes assessment of blood flow through the stent graft, as well as outside of it, renal and iliac blood flow, the maximum diameter of the aneurysm and the presence of flow in a collateral vessel. Convex probes are used to evaluate a larger area using a lower frequency and therefore with greater depth and lower resolution. The probes that provide these conditions have a frequency between 1 and $8 \mathrm{MHz}$. For the visualization of the abdominal aorta, probes are used with a frequency of $2 \mathrm{MHz}$ to $4 \mathrm{MHz} .{ }^{31,32}$ Due to the aneurysm anatomy and the situation of endoleaks, a thorough examination is necessary and requires many important aspects in the evaluation of the aneurysmal sac:

1) A satisfactory B-mode image of the aneurysmal sac and the stent;

2) A satisfactory Echo Doppler image with appropriate gains;

3) A complete study by Echo-Doppler of the entire aneurysm sac outside the graft in a longitudinal and transverse plane for the visualization of endoleaks.

Endoleak surveillance should be conducted with an appropriate and sensitive scale enough to detect slow flow channels. Endoleaks can be distinguished by their artefacts, its reproducibility, uniform nature and their persistence during diastole. The sample spectrum for the non-pulsed Doppler flow stent is used to document the flow direction. Some low-flow endoleaks can be identified as a small colored point at the level of the stent wall during diastole. Reproducibility and persistence allow the differentiation of these leaks regarding artefacts reported.

A Power Doppler (PD) instrument can be helpful when the proper angle of insonation is difficult to achieve with certainty, because it is insensitive to angle considerations. Echo-doppler or PD can aid in the acquisition of a Doppler waveform for the flow within a suspected endoleak to analyse the direction of flow, to provide an estimate of the "resistance" of the endoleak to a nearby collateral vessel. ${ }^{18}$

\section{Conclusion}

Although CT angiography is considered the gold standard for early detection of endoleaks, this technique has some disadvantages which Doppler ultrasound solves effectively. This technique is inexpensive, easy to interpret and implement, with no exposure to any type of radiation, thus reducing the risk of toxicity to the body. Also, it presents comparable quality to CT angiography in relation to the aneurysm measurement and identification of endoleaks after TEVAR. Adding to these advantages the fact that it is a common technique in any vascular laboratory, this will apply to its use in the follow-up of patients after TEVAR implementation..$^{22,25}$

\section{Acknowledgments}

None.

\section{Conflicts of interest}

Authors declare that there is no conflict of interest.

\section{Funding}

None.

\section{References}

1. Nelson BP. Thoracic Aneurysm Clinical Presentation. USA Medscape; 2013.

2. Valentin Fuster, John Willis Hurst. Hurst's the heart. $11^{\text {th }}$ edn. New York, USA: Mc Graw Hill, Medical Pub. Division; 2004. pp. 2472.

3. Nathan DP, Xu C, Pouch AM, et al. Increased wall stress of saccular versus fusiform aneurysms of the descending thoracic aorta. Ann Vasc Surg. 2011;25(8):1129-1137. 
4. Criado FJ. Aneurysm Morphology Matters:Fusiform vs. Saccular. J Endovasc Ther. 2013;20(2):207-209.

5. Aortic Aneurysm Fact Sheet. Centers for Disease Control and Prevention. USA. 2011.

6. Braunwald E, Zipes DP, Libby P. Braunwald's cardiologia: el libro de medicina cardiovascular. Madrid Marban. 2004. pp. 2888.

7. Isselbacher EM. Thoracic and abdominal aortic aneurysms. Circulation. 2005;111(6):816-828.

8. Ramanath VS, Oh JK, Sundt TM, et al. Acute aortic syndromes and thoracic aortic aneurysm. Mayo Clin Proc. 2009;84(5):465-481.

9. Beers MH, Porter RS. The Merck manual of diagnosis and therapy Whitehouse Station: Merck Research Laboratories, Division of Merck \& Co. Inc; USA. 2006.

10. Grabenwoger M, Alfonso F, Bachet J, et al. Thoracic Endovascular Aortic Repair (TEVAR) for the treatment of aortic diseases: a position statement from the European Association for Cardio-Thoracic Surgery (EACTS) and the European Society of Cardiology (ESC), in collaboration with the European Association of Percutaneous Cardiovascular Interventions (EAPCI). Eur Heart J. 2012;33(13):1558-1563.

11. Hoang JK, Martinez S, Hurwitz LM. MDCT angiography of thoracic aorta endovascular stent-grafts: pearls and pitfalls. AJR Am J Roentgenol. 2009;192(2):515-524.

12. Kobeiter Kobeiter H, Nahum J, et al. Zero-contrast thoracic endovascular aortic repair using image fusion. Circulation. 2011;124(11):e280-e282.

13. White GH, Yu W, May J, et al. Endoleak as a complication of endoluminal grafting of abdominal aortic aneurysms: classification, incidence, diagnosis, and management. J Endovasc Surg. 1997;4(2):152-168.

14. Fattori R, Napoli G, Lovato L, et al. Descending thoracic aortic diseases:stent-graft repair. Radiology. 2003;229(1):176-183.

15. Morales JP, Greenberg RK, Lu Q, et al. Endoleaks following endovascular repair of thoracic aortic aneurysm:etiology and outcomes. $J$ Endovasc Ther. 2008;15(6):631-638.

16. Robert J, Rosen MD, Jacqueline T. Endoleaks: a persistent problem. $O R$ Nurse. 2009;3(5):5-56

17. Ashoke R, Brown LC, Rodway A, et al. Color duplex ultrasonography is insensitive for the detection of endoleak after aortic endografting :A Systematic Review. J Endovasc Ther. 2005;12(3):297-305.

18. Heilberger P, Schunn C, Ritter W, et al. Postoperative color flow duplex scanning in aortic endografting. J Endovasc Surg. 1997;4(3):262-271.

19. Chagas Neto FA das, Barreto ARF, Reis HF dos, et al. A importância do diagnóstico por imagem na classificação dos endoleaks como complicação do tratamento endovascular de aneurismas aórticos. RAM Revista de Administração Mackenzie. 2010;43(5):14-25.
20. San Norberto EM, Taylor J, Vaquero C. Color doppler ultrasonography in the monitoring of endovascular abdominal aortic aneurysm repair. In: Grundmann R, editor. Diagnosis and treatment of abdominal and thoracic aortic aneurysms including the ascending aorta and the aortic arch. Spain: Valladolid University Hospital; 2011. pp. 38-56.

21. Agarwal PP, Chughtai A, Matzinger FR, et al. Multidetector CT of thoracic aortic aneurysms. Radiographics. 2009;29(2):537-552.

22. Parmer SS, Carpenter JP, Stavropoulos SW, et al. Endoleaks after endovascular repair of thoracic aortic aneurysms. J Vasc Surg. 2006;44(3):447-452.

23. Pitton MB, Schweitzer H, Herber S, et al. MRI versus helical CT for endoleak detection after endovascular aneurysm repair. AJR Am J Roentgenol. 2005;185(5):1275-1281.

24. Wolf YG, Johnson BL, Hill BB, et al. Duplex ultrasound scanning versus computed tomographic angiography for postoperative evaluation of endovascular abdominal aortic aneurysm repair. J Vasc Surg. 2000;32(6):1142-1148.

25. John Camm A, Luscher Thomas F, et al. European Society of Cardiology. The ESC textbook of cardiovascular medicine. $2^{\text {nd }}$ edn. Oxford; Oxford University Press; 2009. pp. 1180-1181.

26. Giannoni MF, Palombo G, Sbarigia E, et al. Contrast-Enhanced Ultrasound Imaging for Aortic Stent-Graft Surveillance. J Endovasc Ther. 2003;10(2):208-217.

27. Schmieder GC, Stout CL, Stokes GK, et al. Endoleak after endovascular aneurysm repair:Duplex ultrasound imaging is better than computed tomography at determining the need for intervention. J Vasc Surg. 2009;50(5):1012-1017

28. Crimi E, Lee JT, Dake MD, et al. Transesophageal echocardiography guidance for stent-graft repair of a thoracic aneurysm is facilitated by the ability of partial stent deployment. Ann Vasc Surg. 2012;26(6):861. e7-861.e9.

29. Van der Starre P, Guta C, Dake M, et al. The value of transesophageal echocardiography for endovascular graft stenting of the ascending aorta. $J$ Cardiothorac Vasc Anesth. 2004;18(4):466-468.

30. Eggebrecht H, Plicht B, Kahlert P, et al. Intramural Hematoma and Penetrating Ulcers:Indications to Endovascular Treatment. Eur J Vasc Endovasc Surg. 2009;38(6):659-665.

31. Carmody K, Moore CL, Feller-Kopman DJ. Handbook of critical care and emergency ultrasound. New York, USA: Mc Graw-Hill Medical; Probe Selection, Machine Controls and Equipment; 2011. p. 25-28.

32. Julio F, Sergio C, Sergio P, et al. Adamastor. Ultra-Sonografia vascular. Revinter: Rio de Janeiro; 2000. p. 148. 